Man and Nature

MAN AND NATURE

L'homme et la nature

\title{
La physiologie des Lumières
}

\section{François Duchesneau}

Volume 2, 1984

URI : https://id.erudit.org/iderudit/1011819ar

DOI : https://doi.org/10.7202/1011819ar

Aller au sommaire du numéro

Éditeur(s)

Canadian Society for Eighteenth-Century Studies / Société canadienne d'étude du dix-huitième siècle

ISSN

0824-3298 (imprimé)

1927-8810 (numérique)

Découvrir la revue

Citer cet article

Duchesneau, F. (1984). La physiologie des Lumières. Man and Nature / L'homme et la nature, 2, 139-156. https://doi.org/10.7202/1011819ar
Résumé de l'article

Using a method which emphasizes the importance of the explanatory systems of the past for the history of science, this article studies eighteenth-century theories of irritability and muscular contraction. The main focus is on Albrecht von Heller and the way his theories are analogous to Newtonian "principles."
Copyright (C Canadian Society for Eighteenth-Century Studies / Sociéte canadienne d'étude du dix-huitième siècle, 1984
Ce document est protégé par la loi sur le droit d'auteur. L'utilisation des services d'Érudit (y compris la reproduction) est assujettie à sa politique d'utilisation que vous pouvez consulter en ligne.

https://apropos.erudit.org/fr/usagers/politique-dutilisation/ 


\title{
LA PHYSIOLOGIE DES LUMIERES
}

\author{
François Duchesneau
}

Mes collègues de la Société canadienne d'étude du $18 \mathrm{e}$ siècle m'ont fait l'honneur de me demander d'ouvrir ce congrès en faisant état des recherches qui ont donné lieu à la publication de mon livre La Physiologie des Lumières: Empirisme, modèles et théories. ${ }^{1}$ La tâche que les organisateurs m'ont confiée est doublement ardue.

En premier lieu, l'auteur est en mauvaise posture lorsqu'on lui demande d'interpréter son oeuvre. Le discours vient à peine de s'achever; et il faudrait qu'il se transformât sur le champ en méta-discours, sous forme de compte-rendu, d'analyse, d'étude critique. Vous admettrez sans doute que la distance critique me fait totalement défaut pour interpréter La Physiologie des Lumières. En second lieu, l'auteur peut-il se résumer? Puis-je vous exposer avec assez de concision une recherche dont l'objet est le devenir détaillé des théories physiologiques pour tout le l8e siècle, depuis Georg Ernst Stahl jusqu'à Xavier Bichat? Dans bien des cas les textes sont peu connus, ou d'abord difficile pour des non-spécialistes: ils supposent des analyses minutieuses sans appui possible sur une littérature secondaire adéquate. Et il s'agissait de "reconstruire" la signification de théories scientifiques: démarche complexe où il faut tirer parti de la philosophie de la science, sans pour autant dénaturer la matière historique dans laquelle s'est façonné tel savoir scientifique particulier. Il faut aussi reconnaitre qu'une discipline en gestation est moins facile à circonscrire qu'une discipline dûment constituée. Tout compte fait, il est plus aisé d'écrire l'histoire de la physique ou de la mathématique au siècle des Lumières que celle d'une discipline au destin tumultueux, mal dissociée de la médecine pour une part, de l'histoire naturelle pour une 
autre, sans cesse impliquée dans les thèses et les antithèses de la philosophie naturelle, et pourtant essentielle à la dynamique de l'Aufklärung, comme en témoigne Maupertuis, ou Buffon, ou Haller, ou Diderot.

Le philosophe que je suis, a retenu la leçon de Claude Bernard: qu'il n'y a pas de connaissance objective des généralités. Si je dois commencer par quelques généralités, il ne saurait s'agir là d'un véritable objet d'analyse. Aussi vais-je accorder le plus clair de mon développement à la doctrine novatrice d'Albrecht von Haller sur la question de l'irritabilité et de la sensibilité. Si je ne faillis pas à la tâche, vous en verrez surgir les principes d'une théorie dominante, celle qu'exposent les Elementa physiologiae corporis humani (1757-1766).

Les résultats que je soumets à votre appréciation, proviennent de recheeches sur la genèse et les modifications de la théorie physiologique au 18e siècle. Je n'ai eu nulle tentation d'inscrire en épigraphe du livre la formule magique de l'Esprit des lois: "Prolem sine matre creatam", car certains travaux avaient montré la voie. Je ne mentionnerai que les livres de G. Canguilhem sur La formation du concept de réflexe aux 17 et $18 \mathrm{e}$ siècles $^{2}$ et de J. Roger sur Les sciences de la vie dans la pensée française du 18e siècle, ${ }^{3}$ les travaux allemands de Rothschuh sur Hoffmann et de Toellner sur Haller. ${ }^{4}$ Mais l'analyse que j'ai poursuivie est spécifique: il s'agissait de faire ressortir les problèmes conceptuels qui forment la trame des théories relatives au fonctionnement de l'être vivant.

La plupart des recherches d'histoire des sciences, particulièrement en pays anglo-saxons, privilégient une conception de la connaissance scientifique: selon cette conception, les problèmes empiriques et les faits d'exérience délimitent le champ des découvertes et celui de l'analyse historique. Par contraste, les recherches qui visent à reconstituer des systèmes explicatifs du passé sont généralement reléguées au second plan. Or, un bref examen des doctrines physiologiques du $18 \mathrm{e}$ siècle révèle que pour résoudre les problèmes empiriques, le tissu des énoncés scientifiques est assez distendu. Ces énoncés se trouvent accommodés à diverses hypothèses: les mêmes faits, par exemple les régénérescences du polype étudiées par Abraham Tembley de Genève servent d'arguments (après 1744) à des explications antinomiques si l'on compare terme à terme les propos des préformationistes et des épigénétistes, des vitalistes et des organicistes. Bref, on assiste à une prolifération de constructions théoriques. J'ai refusé de tenir cette surdétermination des concepts par rapport à leurs référents observationnels pour la fantaisie, le roman métaphysique d'une pré-histoire de la science biologique, qui verrait le jour au $19 \mathrm{e}$ siècle seulement. J'ai préféré supposer que les constructions théoriques, ce que j'appellerai le travail du concept, constituent une activité de connaissance scientifique, moyennant des exigences propres de validation empirique plus ou moins indirecte. Il y a chez Stahl ou Haller ou C.F. Wolff un jeu de l'argumentation, une réflexion sur la méthode qui sont parties intégrantes de la recherche physiologique. J'ai essayé d'ordonner le foisonnement des concepts théoriques suivant les options qui se font jour successivement quant à l'orientation de la recherche.

Je ferai ici allusion à une doctrine contemporaine de philosophie: la méthodologie des programmes de recherche scientifique d'Imre Lakatos.5 
Lakatos part du paradoxe de Duhem-Quine. L'intelligibilité et la validité d'un énoncé de loi scientifique sont toujours fonction d'un contexte théorique. De ce fait, le paradoxe affirme qu'aucun énoncé scientifique ne peut être infirmé isolément par une proposition énonçant un fait d'expérience contraire. Mais si les systèmes scientifiques sont en soi irréfutables, comme programmes de recherche ils s'avèrent susceptibles de révision critique; ils viennent s'inscrire dans des stratégies d'évaluation. Les systèmes scientifiques doivent témoigner de leur capacité de résorber à moyen et long terme les anomalies (les problèmes empiriques non résolus par les systèmes concurrents), et surtout ils doivent démontrer leur capacité à fournir de nouvelles implications vérifiables. Or de tels programmes de recherche reposent sur des postulats heuristiques. Les uns, formant l'heuristique négative, seraient des sortes de présuppositions ontologiques admises comme principes pour construire des hypothèses, et ils sont d'emblée protégés de toute réfutation. Exemples: les trois lois de la dynamique de Newton et le principe de la gravitation pour la physique du $18 \mathrm{e}$ siècle; l'hypothèse corpusculaire pour la chimie de Lavoisier. L'heuristique positive spécifie une méthodologie pour construire des modèles; il ne s'agit pas de règles formelles de méthode, comme si un discours de la méthode était encore possible; il s'agit d'une méthodologie d'utilisation rigoureuse des analogies. L'exemple par excellence était fourni par la série des modèles géométriques et dynamiques utilisés par Newton pour démontrer un système mécanique planétaire congruent aux phénomènes. Représentations figurées de l'objet qu'il s'agit de comprendre et d'analyser, les modèles peuvent être de deux types: respectivement mathématique et iconique; la théorie les utilise de façon inversement proportionnelle suivant le degré de formalisation possible et les techniques de mesure et de calcul disponible. Les schèmes de représentation impliquent sans doute une gradation continue entre les deux types. Quoi qu'il en soit, l'heuristique de la physiologie des Lumières va se constituer sur des systèmes d'analogies explicatives dont le rôle ne saurait être sous-estimé, même si ces systèmes restent très en-deçà de la modélisation mathématique. A l'évidence, dans nombre de cas, ces modèles vont se structurer par imitation des principes explicatifs dans la physique de Newton. Ne pourrait-on dire alors que l'architectonique des hypothèses repose sur une philosophie newtonnienne de la nature (qui en fournirait la postulation non-infirmable)? Je crois que ce serait une thèse outrée, car il n'y a pas de philosophie newtonienne de la nature apte à jouer un rôle analogue à celui de la physique de Descartes. La cosmologie mécaniste est en ruine, et aucun système de représentation métaphysique, de l'ordre du mécanisme, ne peut avoir cours à partir du moment où les Philosophiae naturalis principia mathematica de Newton définissent un statut nouveau, empiriquement inférentiel de la théorie physique. Le siècle sera empiriste pour sa conception de la connaissance objective. Et c'est pourquoi les présupposés théoriques qui encadrent la vis insita de Haller, l'active sentient principle de Whytt, les molécules organiques de Buffon, la vis essentialis de C.F. Wolff, voire le Bildungstrieb de Blumenbach ou le principe vital de Barthez, et qui figureraient dans l'heuristique, ne sont jamais de pures hypothèses a priori. Ces présupposés ont en quelque sorte des essences nominales de Locke (notions en principe abstraites de l'expérience en vue de classer les données d'expérience) par contraste avec des essences réelles inférées d'une métaphysique de la nature. 
J'ai utilisé à mainte reprise dans mon texte la formule "épistémologie newtonienne" pour qualifier cette démarche en vue de justifier leurs postulats que plusieurs physiologistes du $18 \mathrm{e}$ siècle entreprennent, comme pour circonscrire leurs énoncés théoriques à l'intérieur des possibilités de l'expérience. De ce point de vue, la critique est interne à l'heuristique de la science physiologique au $18 \mathrm{e}$ siècle; nous irions jusqu'à dire qu'elle en est l'articulation dominante. D'où, par implication, il serait sans doute utile de réformer certaines notions dogmatiques du prétendu "paradigme newtonien" en prenant ces faits en compte.

Sur cette question de la critique théorique, nous aurions pu faire référence à quelques thèses significatives du Traité des systèmes. Traitant des abus des systèmes abstraits, Condillac élabore des éléments de critique à l'égard des hypothèses. Il établit: 1) qu'il ne suffit pas qu'une hypothèse s'avère conforme à certains faits (raisons a posteriori) pour que sa validité soit attestée ipso facto, compte tenu de l'éventuelle incapacité explicative de termes abstraits sans signification déterminée; 2) qu'il ne suffit pas non plus qu'une hypothèse s'énonce suivant des raisons a priori déterminées, s'il est alors impossible de concevoir comment elle se rattache aux faits à expliquer, lesquels requièrent une analyse complète. En droit donc, l'hypothèse adéquate impliquerait des termes tout à fait déterminés correspondant au déploiement intégral des relations systématiques dans les phénomènes à expliquer. Au système attesté par l'ordre des phénomènes, les philosophes ont indûment substitué un système d'inférences reposant sur des principes abstraits dont les idées n'ont pas été "démêlées". $6 \mathrm{Au}$ double critère négatif de la critique, correspond un double critère positif de la vérité des suppositions: "Pour s'assurer de la vérité d'une supposition, il faut deux choses: l'une de pouvoir épuiser toutes les suppositions possibles par rapport à une question; l'autre, d'avoir un moyen qui confirme notre choix, ou qui nous fasse reconnaitre notre erreur". 7 En définitive, il s'agit d'une double procédure de composition et décomposition des idées. Condillac mentionne la fonctionalité de ce processus d'hypothèse en mathématique; il signale le cas de l'astronomie de position où, moyennant la transposition "géométrique" des directions et des mouvements observés, l'explication hypothétique des "apparences" se trouve analytiquement fondée, mais avec possibilité de modification ultérieure des hypothèses par suite de l'inexhaustion des observations positives. Du point formel, la science des phénomènes naturels est caractéristisée par l'impossibilité d'épuiser toutes les suppositions et par l'absence de critère univoque de choix entre hypothèses. Ces déficiences interdisent d'accorder par exemple à une hypothèse du type de l'hypothèse corpusculaire un statut de représentation adéquate de l'ordre phénoménal, à cause de l'analogie incertaine (non fondée dans les faits) entre l'inobservé et l'observable. Nous n'avons pas moyen de pénétrer les "ressorts" de l'ordre phénoménal par delà les phénomènes: "Or cette ignorance nous laissera dans l'impuissance de remonter aux vraies causes qui produisent et lient, en un seul système, le petit nombre de phénomènes que nous connaissons". 8 Au système des hypothèses cartésiennes, Condillac oppose le système newtonien, dont les énoncés théoriques seraient entièrement analysables en termes d'observation, d'analogie empiriquement vérifiable et de calcul.9 Si l'attraction semble renvoyer à une "qualité occulte", c'est par suite d'une lecture de l'explication suivant le modèle de concepts métaphysiques signifiant l'essence réelle au-delà des phénomènes. A cette 
lecture, Condillac substitue une lecture idéogénétique renvoyant à l'enchaînement des faits dans l'ordre où ils s'entre'expliquent par corrélation des constats empiriques:

L'expérience en nous faisant voir quelques faits qui s'expliquent par d'autres, nous donne un modèle de la manière dont une hypothèse devrait rendre raison de tous. Ainsi pour s'assurer de la bonté d'une supposition, il $n^{\prime} y$ a qu'à considérer si les explications qu'elle fournit pour certains phénomènes, s'accordent avec celles que l'expérience donne pour d'autres; si elle les explique tous sans exception, et s'il n'y a point d'observations qui ne tendent qu'à la confirmer. Quand tous les avantages s'y trouvent réunis, il n'est pas douteux qu'elle ne contribue au progrès de la physique. 10

Si Condillac, comme Locke, accorde aux hypothèses physiques un certain rôle de résumé classificatoire et donc économique des données d'expérience, il attribue à ces hypothèses, lorsqu'elles épousent le modèle méthodologique illustré par Newton, une fonction épistémologique plus rentable, celle d'articuler l'explication à partir des données d'observation en direction d'une pluralité d'autres faits, et ce en vertu du rapport factuel illustré par telle ou telle expérience...

Il n'y a pas de formule unique et univoque pour englober la physiologie des Lumières. Sans perdre de vue la forme prégnante de l'"épistémologie newtonienne", il fallait tenir compte de la variété des modèles et des théories, qui naissent dans certains cas à distance de la science newtonienne proprement dite. J'ai donc privilégié un examen suffisamment descriptif, suffisamment analytique pour permettre de faire ressortir la cohérence propre et les astuces épistémologiques diverses des théories qui ont déterminé la genèse et l'évolution de la physiologie. Fait caractéristique: le passage d'une théorie à l'autre ne résulte jamais d'une infirmation en règle. Les théories par ailleurs se recoupent, mais il semblerait bien difficile de décrire des superpositions possibles permettant des réductions au sens logique. Certains énoncés de fait fournissent à coup sûr des motifs de validation: ils proviennent, entre autres, d'observations sur les phénomènes de contraction et de sensibilité des fibres, sur les stades de développement de l'embryon, sur les réactions de l'animal spinal ou sur le polype morcelé et reconstitué. Mais aucune de ces expériences n'est cruciale au sens technique du terme. De Stahl à Bichat, nous assistons sans doute à une invention d'options théoriques qui permettent à l'explication physiologique de s'affirmer suivant des stratégies de recherche tendant sans cesse à une plus grande pertinence.

Le 22 avril et le 6 mai 1752 Haller présente les deux parties d'un mémoire sur la sensibilité et l'irritabilité à la Société royale des sciences de Göttingen. Le tout est publié une première fois en 1752 , sous le titre De partibus corporis humani sensilibus et irritabilibus, 11 puis traduit en français par le docteur Tissot en 1755 sous le titre Dissertation sur les parties irritables et sensibles des animaux. 12 Enfín à partir de 1756 paraissent les Mémoires sur la nature sensible et irritable des parties du corps animal où se trouvent regroupés la Dissertation, les éclaircissements, les textes de divers auteurs sur les phénomènes étudiés; et les 
réponses aux objections.13 En 1751, l'élève de Haller, Johann Georg Zimmermann avait lui-même publié sa Dissertatio physiologica de irritabilitate où il exposait certains résultats des recherches entreprises sous la direction du maitre. Quant à Haller, il faisait déjà mention de la nature irritable dès 1739 dans son commentaire des Institutiones de Boerhaave; il y faisait allusion dans les Primae lineae physiologiae de 1747.14 En fait, il se consacrait de 1746 à 1752 aux expériences concluantes. Le texte de la Dissertation sur les parties irritables et sensibles des animaux est au coeur de la théorie physiologique de Haller. Les concepts et les expériences qui s'articulent autour du thème de l'irritabilité et de la sensibilité peuvent seuls expliquer les principales doctrines des Elementa physiologiae corporis humani (1757-1766), c'est-à-dire du premier grand traité de physiologie de l'histoire moderne.

Le concept d'irritabilité avait fait son apparition dans la doctrine physiologique de Francis Glisson (1597-1677). Haller, qui se réfère au De ventriculo \& intestinis (1677), attribue à Glisson la thèse que la force vive des éléments du corps en général doit être rapportée à une perception naturelle non-consciente, sous la dépendance de l'archée fabricatrice du corps propre. La perception en question se rattache d'une part aux sens externes, d'autre part à une sorte d'appétit interne. L'irritabilité est le pouvoir qui correspond à une telle activité de perception. Glisson détache l'irritabilité de la perception consciente, en signalant la subsistance d'activité contractile dans les parties après la mort. Mais, si Haller accepte une telle distinction, il reproche à Glisson d'avoir lié l'irritabilité à toute forme de contraction morte. Pour autant que le médecin anglais ait eu raison de distinguer force nerveuse et force d'irritabilité, il se livre à une généralisation indue en voulant attribuer le pouvoir d'irritabilité à d'autres parties, les os et les liquides vitaux, par exemple, plutôt qu'aux seules fibres musculaires.

Parmi les iatromécaniciens italiens, Lorenzo Bellini (1643-1704), dans De stimulis (1683) et le De missione sanguinis (1683), avait posé l'existence d'une force contractile naturelle par laquelle s'expliquerait l'expulsion des substances âcres, lorsqu'elles viennent irriter les fibres. Sous l'action des irritants, cette force ferait mouvoir mécaniquement les muscles, accélérerait le mouvement du sang et interviendrait pour produire les phénomènes d'inflammation, de révulsion, de dérivation et d'évacuation. Cette hypothèse ne reposait en fait sur aucune recherche d'expérience. D'un point de vue plus expérimental, Giorgio Baglivi (1668-1707), dans le De fibra motrice et morbosa (1703), avait montré l'existence d'un mouvement de constriciton de la fibre cardiaque dans le coeur extirpé: phénomène qu'il rattachait à un processus chimique dû à la circulation des esprits animaux. 15 Or, par une tendance antinomique à celle que représentait la doctrine glissonienne, les iatromécaniciens tendent à rattacher l'irritabilité à la force nerveuse même, en la faisant dépendre d'une structure des fibres de type vasculaire suivant le modèle des nerfs. La position de Boerhaave est caractéristique:

[il] a reconnu une force active dans le coeur, et un principe caché de mouvement dans ses morceaux coupés; mais son système sur la cause du mouvement des muscles, qu'il attribuait aux nerfs, prouve qu'il n'a point connu assez manifestement, que la cause de ce mouvement 
était dans les muscles mêmes, que les nerfs n'avaient d'autres fonctions, que de l'augmenter au gré de l'âme, et qu'ils pouvaient bien l'augmenter ou le diminuer, mais qu'ils n'en étaient point la cause, puisqu'elle a une étendue bien plus vaste que les nerfs, et qu'on la trouve dans des insectes, qui n'ont pas même de tête.16

Haller met ainsi en cause la méconnaissance par les iatromécaniciens de la propriété spécifique des fibres musculaires.

D'ailleurs, le même type de conceptualisation générale freine la reconnaissance de l'irritabilité chez G.E. Stahl (1659-1734) et ses disciples. Dans ce cas également, l'ordre prêté à l'organisme est global et unitaire et n'admet pas de propriétés organiques spécifiques. Les stahliens en effet font intervenir la tonicité comme disposition fonctionnelle des fibres, capable de rendre compte de leur contraction naturelle; mais, comme toutes les opérations vitales, la tonicité est liée, en tant que mode même d'opérations, à l'organisme, c'est-à-dire au mécanisme corporel dans la mesure où il est subordonné à un ordre téléologique: c'est donc l'âme qui est le principe actif et la raison suffisante des phénomènes relevant de l'irritabilité. Stahl reconnait que le mouvement nerveux s'ajoute à ce que nous pourrions appeler la tonicité de base, et de ce point de vue, il se rapproche de la conception hallérienne des forces différentielles de l'activité musculaire, mais il étend la tonicité à toutes les parties anatomiques et il étaie cette généralisation sur le principe de l'intervention hégémonique de l'âme dans l'ensemble de l'organisme.

Haller relève le fait que même chez ses contemporains immédiats, dont les travaux et les expériences corroborent ses propres recherches, la conception d'un système structuro-fonctionnel unitaire met en cause la thèse à laquelle il tient surtout, celle de la délimitation du principe d'irritabilité comme principe particulier à la fonction des fibres musculaires. C'est ainsi que Gorter (1689-1762) dans le De motu vitali (1751) attribue la force vitale à toute fibre comme propriété commune. Quant à F. Winter (1712-1760), s'il fait de l'irritabilité le principe du mouvement vital musculaire, c'est pour en situer le siège dans le réseau nerveux et pour assigner à l'irritation même des esprits le phénomène moteur consécutif.17 Le cas de Robert Whytt dans son Essay on the Vital and Other Involuntary Motions of Animals (1751) est également significatif puisque Whytt pèche par le même défaut théorique que les iatromécaniciens, l'excès dans la généralisation structuro-fonctionnelle, alors qu'il maintient une forme d'animisme pour l'explication particulière des mouvements vitaux dans les organes extirpés:

... et depuis peu, affirme Haller, le célèbre M. Whytt attribue tous les mouvements du corps humain à la force du stimulus: avec cette différence, entre lui et les Auteurs que je viens de nommer (d'allégeance iatromécaniste), qu'il attribue l'irritabilité à l'âme, qui, sentant l'impression de l'irritation, occasionne la contraction de la fibre. 18

Haller dénonce le peu de cohérence entre les données expérimentales recueillies par Whytt et l'échafaudage théorique auquel il se livre dans la foulée de l'animisme de Stahl et de Boissier de Sauvages (1706-1767). Le 
nouveau matérialisme, celui qui prendra bientôt son essor avec Diderot et d'Holbach et aboutira aux positions spéculatives de l'idéologie chez Cabanis, s'annonce avec $L$ 'homme machine (1747) de La Mettrie (1709-1751). Or La Mettrie lui aussi trahit, au dire de Haller, la spécificité du principe d'irritabilité en arguant que l'irritabilité, comme propriété des fibres, illustre un dynamisme de la matière organisée susceptible d'expliquer tous les phénomènes que nous faisons dépendre du contrôle et de l'action d'un agent propre de sensibilité. Haller se défend d'avoir été le père d'un système aussi impie, auquel seule la rouerie de La Mettrie, ancien élève de Boerhaave comme lui, a réussi à lier son nom. 19 Par contraste, l'essentiel de la nouvelle théorie de Haller, du point de vue épistémologique, c'est la distinction des propriétés vitales en fonction des structures organiques dont elles dépendent et en fonction des phénomènes qui en spécifient le mode d'opération. Ceci nous amène à l'examen de la thèse de Haller.

A cet égard, mon objectif sera de montrer: 1) ce que signifie la méthodologie dite "newtonienne", mise en oeuvre par Haller dans l'analyse de l'irritabilité; 2) comment la jonction s'opère sur ce point entre la théorie et la pratique expérimentale. A partir des interprétations, en quelque sorte opposées, de R. Toellner 20 et de T.S. Hall, 21 je chercherai à établir le statut épistémologique de l'irritabilité comme analogue d'un principe au sens de Newton.

Comme propriétés de certaines parties de l'organisme, l'irritabilité et la sensibilité se trouvent définies par Haller conformément aux critères de l'expérience qui permettent de les identifier et de les distinguer:

\begin{abstract}
J'appelle partie irritable du corps humain, celle qui devient plus courte, quand quelque corps étranger la touche un peu fortement. En supposant le tact externe égal, l'irritabilité de la fibre est d'autant plus grande, qu'elle se raccourcit davantage... J'appelle fibre sensible dans l'homme, celle qui étant touchée, transmet à l'âme l'impression de ce contact: dans les animaux, sur l'âme desquels nous n'avons point de certitude, l'on appellera fibre sensible, celle dont l'irritabilité occasionne chez eux des signes évidents de douleur et d'incommodité. 22
\end{abstract}

Haller a en vue de récuser l'extension abusive de telle ou telle propriété par le jeu de l'analogie à des parties qui ne la manifestent pas directement à l'expérience. La définition se veut empirique et donne lieu à un essai de quantification. Haller critique son maitre Boerhaave pour avoir attribué la sensibilité et un certain mouvement correspondant à toutes les parties du corps humain sous le couvert de sa théorie de la structure organique et en conformité avec le modèle vasculaire, qui ramène de façon générale toutes les membranes à une texture de petites canalisations nerveuses. 23 La définition des propriétés organiques ne peut reposer de la sorte sur une hypothèse de structure procédant à la généralisation extrême d'un type particulier d'organisation.

Toutefois, c'est dans un cadre théorique bien arrêté que Haller institue ses distinctions phénoménales et empiriques. Les faits d'expérience 
prennent la signification qu'il leur donne, par l'effet d'une systématisation plus ou moins implicite des mouvements vitaux en relation aux structures organiques et aux types de dispositifs dynamiques qu'elles représentent. La théorie de l'irritabilité doit permettre de concevoir l'inhérence d'une propriété à la structure spécifique de la fibre musculaire sous forme de pouvoir spécifique, et de concevoir pareillement la relation qui lie les phénomènes comme effets à ce type de structure ou de dispositif moteur. Le même cadre théorique rattachera la sensibilité à un processus causal plus indirect, déterminé par l'impression sensible: le processus implique alors la transmission à travers le réseau nerveux et la réceptivité centrale des sensations, lesquelles peuvent à leur tour se transformer en phénomènes moteurs.

La distinction fonctionnelle entre sensibilité et irritabilité repose sur la distinction des forces qui interviennent dans la contractilité des muscles. Ces forces agissent différemment les unes des autres: elles supposent donc des structures causales différentes. D'abord, Haller distingue les propriétés vitales comme telles de l'élasticité, simple propriété physique des fibres. L'élasticité est la force contractile la plus faible; elle subsiste tant que la fibre conserve sa structure après la mort, et jusqu'à son entière dissolution; elle se manifeste seulement si les fibres ont été tendues ou comprimées; elle n'appartient pas de façon exclusive aux fibres musculaires, mais à toutes les fibres qui sont dotées de flexibilité. Cette propriété qui n'a rien de commun avec la vie, fait que les fibres, quand on a coupé les muscles, se retirent vers les parties solides auxquelles elles sont attachées. 24 La seconde force naturelle aux muscles est vitale: elle subsiste et se manifeste tant que les fibres ne sont pas ankylosées dans leurs mouvements par les effets de la mort; elle persiste dans les parties musculaires soustraites à toute intervention de la sensibilité; ce qui la caractérise, c'est le mouvement alternatif de contraction et de décontraction sous l'effet d'une excitation, qui peut être ou non d'origine nerveuse; on la trouve tant dans les animaux qui sont dépourvus de nerfs que chez les animaux innervés dans le coeur, les intestins, les muscles divers, séparés du corps. Haller insiste sur le fait que les muscles en sont le siège par excellence vu la structure spécifique de leur fibre et l'originalité des phénomènes d'irritabilité par rapport à la simple contractilité élastique. 25 La troisième force a une action élective et sporadique, dépendant de l'excitation des nerfs par l'impression sensible ou par la détermination volontaire. Son action se traduit par un accroissement de contractilité vitale, ou par une opération coordonnée de réaction aux stimuli. Il s'agit de la sensibilité. Surtout, la sensibilité périt lorsqu'on a lié ou sectionné le nerf qui parvient normalement au muscle; la force d'irritabilité subsiste au-delà, dans les parties devenues insensibles mais possédant encore les conditions de flexibilité compatibles avec l'exercice d'une telle force.

Un autre support théorique de la distinction entre sensibilité et irritabilité est la conception fibrillaire des structures organiques. Aux muscles appartient, du fait de leur structure fibrillaire, un degré différentiel d'irritabilité, comme propriété déterminant la contraction vitale. En dernier ressort, Haller est en mesure d'établir une loi de proportion structuro-fonctionnelle distincte et pour l'irritabilité et pour la sensibilité. Robert Whytt avait formulé dans son Essay on the Vital and Other 
148.

Involuntary Motions of Animals (1751) une loi de corrélation entre l'intensité des mouvements vitaux et la quantité et la dimension des fibres nerveuses. 26 Du point de vue des résultats expérimentaux, Haller s'inscrit en faux contre cette généralisation qui lui parait abusive. Ce qu'il propose, c'est la double loi de corrélation structure-fonction que voici: "La sensibilité est en même raison que le nombre de nerfs, et leur nudité: au lieu que l'irritabilité est en général en raison du nombre de fibres exposées à la cause irritante".27 Or cette double loi dépend non seulement des données d'expériences recueillies et de la classification utilisée, elle est aussi tributaire de la distinction fonctionnelle de l'agent de la sensibilité par rapport aux agents des autres mouvements vitaux, de la distinction des forces en jeu dans l'activité organique, et du rapport des propriétés à la typologie des structures fibrillaires. Compte tenu de cet exemple privilégié, comment interpréter la méthodologie empiriste mise de l'avant par Haller?

Le processus d'analyse par lequel l'irritabilité est reconnue comme propriété spécifique de la fibre musculaire se signale comme un processus de séparation des phénomènes caractéristiques par rapport aux phénomènes qui semblent requérir une raison explicative analogue, mais qui en fait relèvent de propriétés différentes.

Au moment, explique Haller, où je commençais les expériences, d'une part, mon espoir était de pouvoir dépasser quelque peu les connaissances antérieures, d'autre part, je croyais pouvoir supposer qu'il fallait procéder par soustraction à l'égard de ce que pensaient les savants. J'ai donc séparé la nature irritable de la force morte d'une part, de la force nerveuse d'autre part, et du pouvoir de I'âme. 28

Haller précise les stades de la distinction: 1) mise en évidence du fait que la nature irritable du coeur et des intestins dépend d'une propriété spécifique; 2) réduction de la propriété d'irritabilité à la structure de la fibre musculaire, moyennant la séparation de la force irritable qui appartient en propre aux muscles par rapport à la force contractile commune à toutes les fibres composantes de l'organisme; 3) distinction d'une réceptivité particulière des fibres irritables par rapport aux stimuli, réceptivité provenant elle-même d'un pouvoir autonome de déclenchement du mouvement dans ces fibres; 4) dernier stade: la force vive opérant dans l'irritabilité s'exerce d'elle-même, car souvent nul stimulus indirect d'origine nerveuse n'est requis pour que s'opère le passage immédiat du repos au mouvement; même dans le cas où un stimulus nerveux semble requis, il y a disproportion significative entre la faible intensité de l'excitant et l'effet considérable qui peut en résulter. De discrimination en discrimination, sous réserve d'un contrôle expérimental strict, se trouve isolée la propriété spécifique d'une structure déterminée à l'intérieur de l'organisme. Corrélativement, la théorie se trouve limitée à une fonction explicative empirique. "Deux de mes amis, explique Haller, MM. Zimmermann et Oeder ont suivi la véritable route pour parvenir à connaitre cette propriété; l'expérience leur a appris qu'elle était comme l'attraction, une loi de la nature, et ils ont abandonné des recherches inutiles sur la théorie".29 De même pour les autres expérimentateurs de l'école hallérienne. Reste à savoir évidemment comment s'articule un tel processus de constitution de la théorie et sur quelles justifications épistémologiques Haller pouvait s'appuyer. 
Qu'est-ce qui empêche d'admettre l'irritabilité pour une propriété du gluten animal, tout comme on reconnait l'attraction et la gravité pour propriétés de la matière en général, sans pouvoir en déterminer les causes? Les expériences nous ont appris l'existence de cette propriété, elle a une cause physique sans doute, qui dépend de l'arrangement des dernières parties, mais que nous ne pouvons pas connaitre, parce qu'il ne peut pas être saisi par des expériences aussi grossières que celles auxquelles nous sommes bornés. 30

En principe, tout le processus d'explication de l'irritabilité tient dans cette conjonction d'empirisme et de postulat newtonien. En fait, des principes rationnels particuliers guident le processus explicatif. En premier lieu, Haller a procédé à une analyse de la structure organique qui aboutit à mettre en évidence la structure et la composition de la fibre. Or toute fibre, et non pas seulement la fibre musculaire, possède une certaine quantité de gluten dans sa composition chimique. Il semblerait donc que le principe de déstructuration analytique de l'organisme dût amener la constatation que l'irritabilité est la propriété générale de la fibre vivante. Toutefois, la propriété se traduit par des effets observables, qui correspondent à la seule structure des fibres musculaires. Par rapport au principe newtonien d'attraction, le principe d'irritation a le caractère d'un dispositif fonctionnel spécial et que l'on peut difficilement rapporter aux structures une fois que celles-ci se trouvent analytiquement réduites en leurs éléments: ce principe ne se révèle qu'au niveau de structures déjà relativement complexes, où le gluten peut entrer comme élément matériel constituant, mais non pas être la raison suffisante de la propriété. L'ambiguité du principe à cet égard le différencie quelque peu du principe de l'attraction newtonienne. Le principe newtonien renvoie à une cause explicative suffisante d'un effet tout à fait général des éléments matériels. Dans le dernier "query" de son Opticks, Newton s'était défendu de rétablir, en faisant appel aux principes de la gravitation et de l'attraction, les qualités occultes de la scolastique. Répondant aux critiques de Leibniz et de Huyghens qui lui reprochaient de s'écarter de la seule intelligibilité géométrique au profit d'agents causaux d'un type tout à fait hypothétique, Newton avait distingué qualités occultes spécifiques et principes généraux de mouvement:

To tell us that every species of things is endowed with an occult specific quality by which it acts and produces manifest effects, is to tell us nothing: but to derive two or three general principles of motion from phaenomena, and afterwards to tell us how the properties and actions of all corporeal things follow from these manifest principles, would be a very great step in philosophy, though the causes of those principles were not yet discovered: and therefore I scruple not to propose the principles of motion above-mentioned, they being of very general extent, and leave their causes to be found out. 31

R. Toellner cite ce passage à l'appui d'une explication de la méthodologie hallérienne et il rappelle que Haller justifie à l'occasion ses inférences par références aux principes newtoniens. 32 Mais il s'agit en fait chez Haller de références analogiques parfois très ambiguës. T.S. Hall, dans son article "On Biological Analogues of Newtonian Paradigms," met justement 
en question le parallélisme d'une démarche comme celle de Haller et de la méthodologie de l'explication suivant le modèle de la physique de Newton. Il fait ressortir, d'une part, l'universalité des principes de mouvement par contraste avec la spécificité de certains principes physiologiques, soi-disant analogues, tel le principe d'irritabilité; d'autre part, il fait ressortir qu'un principe newtonian est adéquat aux phénomènes dont il constitue la raison explicative; les lois physiques auxquelles le principe doit sa forme, permettent une détermination générale vérifiable pour tous les phénomènes concernés. Par contraste, les analogues biologiques du principe newtonien ne sont que des propriétés auxquelles l'explication rattache des phénomènes peu quantifiables; de plus de telles propriétés résultent d'une typologie d'observation des phénomènes dont les normes ne laissent pas d'être variables. L'éventail limité des principes newtoniens contraste avec les variantes infinies d'un principe comme celui de l'irritabilité hallérienne au travers des doctrines contemporaines ou subséquentes. Mais Hall laisse dans l'ombre le lien qui unit le modèle explicatif et une forme d'analyse et d'expérimentalisme. Qu'il s'agisse d'attraction ou d'irritabilité, on a désormais affaire à une classe de phénomènes typiques, plus ou moins généralisables, qui servent à analyser, et donc à distinguer, certains processus au sein de phénomènes plus complexes. La quantification des processus moteurs change-t-elle fondamentalement le type d'analyse? Sans doute non, dans la mesure où il s'agit dans les deux cas d'établir une analyse des phénomènes sur la base d'un modèle dont les éléments mêmes sont fournis par l'expérience: les phénomènes sont ainsi susceptibles de servir de normes pour concevoir l'ordre phénoménal et pour vérifier l'explication. Le principe de la réitération des expériences en vue d'éliminer les variables parasites, qui est un dogme de Haller, ressortit à cette théorie nouvelle de l'expérience dont les bases sont proposées par l'empirisme de Locke et illustrées par la méthodologie de l'Experimental Philosophy, y compris chez Newton. Le fondement implicite de la règle est bien l'idée que le phénomène lui-même peut être significatif et servir de norme à l'explication: "Des causes étrangères, précise Haller, peuvent introduire de l'erreur dans des expériences, qu'on ne vérifie pas, mais ces causes se séparent à mesure qu'on réitère le même fait, par là même qu'elles sont étrangères; après un certain nombre de vérifications il ne reste que les résultats, qui naissent nécessairement de la nature des choses." 33 Voilà donc pour l'épistémologie newtonienne de Haller.

Par contre, il est incontestable que le modèle newtonien sert à couvrir une pratique différente et originale de l'explication. Haller rapporte la propriété vitale à une cause physique se réduisant pour finir à l'arrangement des parties internes à la fibre et imperceptibles; en même temps, il se sert de cette imperceptibilité de l'arrangement mécanique et géométrique des éléments matériels pour reporter l'explication au niveau spécifique et plus global des phénomènes physiologiques. La cause inconnaissable sert alors à centrer l'analyse sur un phénomène dont la base structurelle est relativement plus complexe que celle sur laquelle repose la gravitation ou l'attraction-répulsion: il s'agit du phénomène de la contractilité musculaire vive; et ainsi les mouvements fibrillaires et organiques sont expliqués à partir d'une telle propriété. Il en résulte l'analyse hallérienne avec les caractéristiques que nous lui avons reconnues. Mais le principe de la cause inconnaissable intervenant à ce niveau de l'analyse ouvre la possibilité de débats spéculatifs ou théoriques auxquels la physique newtonienne ne donnait pas lieu de la même manière. 
D'où un certain nombre de problèmes spécifiques. Le recours à la cause inconnaissable ainsi articulé à l'explication physiologique ne peut-il permettre d'étendre l'irritabilité aux phénomènes généraux de la motricité organique, et donc à l'ensemble des fonctions vitales? Ne peut-il permettre une spéculation indéfinie sur la distinction de l'organicité et de la sensibilité puisque la sensibilité elle aussi se traduit en effets et phénomènes moteurs? Ne peut-il permettre de concevoir la vie au sein de la matérialité par delà le mécanisme newtonien par avènement ou résurgence ambiguë du vitalisme chez Barthez et chez Blumenbach, ou d'une monadologie physiologique, comme chez Maupertuis, à laquelle la théorie leibnizienne du vivant pourra rétrospectivement servir de modèle.

L'embarras de Haller et l'ambiguité de la théorie s'expriment dans les règles méthodologiques qu'il énonce, en réponse aux hypothèses auxquelles l'irritabilité donne ou peut donner lieu. Le principe de la distinction expérimentale des propriétés structurales et fonctionnelles suivant le type des parties est sa réponse aux nouveaux systèmes de l'"économie animale" qui s'édifient sur l'extension de l'irritabilité à l'ensemble des organes.34 Mais le problème se prolonge au-delà de la séparation des propriétés conformément à une typologie des phénomènes spécifiques. Haller en est conscient: il distingue l'irritabilité et la sensibilité comme propriétés structurales et fonctionnelles, mais rejette au plan de la conjecture le fondement causal de la distinction expérimentale. L'interprétation des causes, même limitée aux données de l'analyse opérée sur les phénomènes, laisse en suspens la détermination même des principes comme distincts: "Cachées vraisemblablement [il s'agit des causes] dans la texture des dernières molécules de la matière, hors de la portée du scalpel et du microscope; tout ce que l'on peut dire là-dessus, se borne à des conjectures, que je ne hasarderai pas..."35 Haller lui-même donne comme naturelle la tendance à étendre l'empire de l'irritabilité et à y rattacher l'explication unitaire des fonctions: mouvements des artères, sécrétions des glandes, phénomènes pathologiques particuliers... C'est l'absence de base expérimentale suffisante pour cette extension qui le ramène à la discrimination méthodique des phénomènes d'irritabilité proprement dite par rapport aux autres phénomènes physiologiques. 36

Et encore il est manifeste que l'effort d'édification théorique auquel l'irritabilité est susceptible de donner lieu, ne peut faire l'objet de contraintes méthodologiques rigoureuses. S'il énonce quelques postulats concernant la structure fibrillaire comme structure organique de base, Haller se garde de toute spéculation intempestive sur la cause interne de l'irritabilité. Toutefois, dans cet état de précaution méthodologique, il n'évite pas parfois de suggérer des modes de conceptualisation dont les conséquences sont susceptibles d'affecter la théorie de l'être vivant dans son ensemble. Dans le recueil des Mémoires, un certain passage des lettres du P. Urbano Tosetti résume Haller et prétend restreindre les perspectives spéculatives qui s'ouvrent à partir du concept d'irritabilité: "... L'on démontre ensuite que les parties les plus irritables ne sont point sensibles, et que les plus sensibles ne sont point irritables: d'où l'on conclut que l'irritabilité ne dépend point des nerfs, mais de la fabrique primordiale des parties". 37 Supposons que la démonstration établisse expérimentalement la différence des parties sensibles et des parties irritables--ce qui semble être le cas--et qu'il soit évident que l'irritabilité ne dépende point des nerfs--ce que l'expérience ne pourrait montrer que 
suivant une exhaustion indéfinie des cas particuliers représentés par les diverses structures anatomiques--, il reste que la raison explicative, recherchée dans "la fabrique primordiale" des parties, relève d'une hypothèse dont les limites sont mal assignées et dont l'intelligibilité est imprécise. Dans la section XV des expériences, qui concerne le mouvement péristaltique de l'estomac et de l'oesophage, Haller éclaire quelque peu cette explicative en affirmant: "Un mouvement ne peut pas être dans le corps humain sans qu'il ait des causes suffisantes dans la structure de la partie, et l'effet ne saurait se déduire sans la cause." 38 Cet axiome lui sert alors à inférer du mouvement de l'estomac, la structure musculaire, seule compatible selon la discrimination expérimentale avec un mouvement qui ne dépend point de la force nerveuse transmise par le réseau de la moelle épinière et des nerfs. Mais il faut voir de façon générale ce qu'un tel axiome "mécaniste" implique pour la théorie de l'irritabilité. Il est sous-entendu que le phénomène d'irritabilité ne serait que la résultante d'un agencement mécanique spécial des parties élémentaires. Mais pas n'importe quel agencement mécanique: le caractère spécial de l'agencement est affirmé en prenant en compte que les effets de l'irritabilité sont hétérogènes par rapport aux phénomènes de l'élasticité et la contractilité morte. La nature et l'ordre spécifique des phénomènes vitaux sont sauvegardés dans le cadre d'une hypothèse mécaniste spéciale. Finalement, ce sont les suggestions tirées de l'expérience qui orientent les distinctions conceptuelles, sans quoi la physiologie serait le terrain vague de toutes les hypothèses.

Haller se sert de l'incapacité à se représenter géométriquement et mécaniquement les propriétés élémentaires des constituants de la fibre vivante, pour reporter l'explication au niveau spécifique et plus global des phénomènes vitaux. Ce type de jonction entre théorie et pratique va permettre la constitution de la doctrine physiologique dominante au siècle des Lumières mais ce type de jonction entre théorie et pratique va aussi laisser le champ libre au developpement d'hypothèses plus vastes, organicistes ou vitalistes, avec apparition éventuelle d'une sorte de monadisme physiologique (sensibilité sourde des parties, propriétés fonctionnelles relativement autonomes des éléments dans le composé organique). Haller refuse de s'engager dans cette voie à cause des principes régulateurs d'une méthodologie empiriste qui entend limiter la fonction explicative des hypothèses à corréler et à distinguer de la façon la plus stricte possible les phénomènes dans le champ de la physiologie. En vertu de la même référence newtonienne, certains de ses successeurs pourront en décider autrement et construire des systèmes vitalistes en invoquant et en intégrant l'irritabilité hallérienne. 
NOTES

1 François Duchesneau, La Physiologie des Lumières: Empirisme, modèles et théories (La Haye: Martinus Nijhoff, 1981). (Archives internationales d'histoire des idées - 95), XXI, 611 pages. Martinus Nijhoff B.V. nous a généreusement autorisé à citer des fragments du chapitre $V$ dans le présent travail.

2 Paris: Presses Universitaires de France, 1955.

3 2e édition. Paris: Armand Colin, 1971.

4 K.E. Rothschuh, "Studien Zu Friedrich Hoffman (1660-1746)", Sudhoffs Archiv für Geschichte der Medizin und der Naturwissenschaften, 60 (1976), 163-193, 235-270. Richard Toellner, Albrecht von Haller: Über die Einheit im Denken des letzen Universalgelehrten (Wiesbaden: Franz Steiner, 1971).

5 Imre Lakatos, The Methodology of Scientific Research Programmes Philosophical Papers, Volume I (Cambridge: Cambridge University Press, 1978).

Condillac, Traité des systèmes, en Oeuvres philosophiques de Condillac (Paris: Presses Universitaires de France, 1947), I, 194.

Condillac, I, 195.

8 Condillac, I, 197.

9 Condillac, I, 200.

10 Condillac, I, 203.

11 Commentarii Societatis Regiae Scientiarum Gottingae ad annum 1752 (Gottingae, 1753), II, 114-158.

12 Lausanne: M.M. Bousquet, 1755.

13 Lausanne: M.M. Bousquet (-S. d'Arnay), 1756-1760. 4 vol. in -8.

14 Cf. Elementa, IV, p. 462: "Ego vero, postquam ad experimenta accessi [De irritabili natura primum dixi anno 1739 in comm. ad Boerh. n. 187, p. 1.2. deinde ann. 1743. in iisdem Comm T. IV p. 586. \& firmius in primis Physiologiae lineis ann. 1747], partim paulo ultra priora speravi me progredi posse, \& partim detrahendum esse aliquid de iis, quae alii $\mathrm{Cl}$. viri putarent, se sibi sumere posse". Cf. Mémoires, I, 3-4: Haller y fait allusion aux expériences faites depuis 1746 et il indique que ces recherches ont donné lieu à une "nouvelle division des parties du Corps humain", en tant qu'elles sont susceptibles ou non d'irritabilité et de sensibilité. 
15 Cf. M.D. Grmek, "La notion de fibre vivante chez les médecins de l'école iatro-physique", Clio Medica, 5 (1970), 297-318.

Mémoires, I, 86-87.

17 Elementa, IV, 462: "Inde, cum experimenta mea innotuissent, subito tanta incrementa cepit irritabilitas, ut omnes omnino vitales \& involuntarii in corpore humano motus $a b$ ea deducerentur". Haller se réfère à ce propos à Winter, Parson, Baker, Cigna, v. Geuns, Andrea, Petrini, Man, Gaubius, Whytt et Krüger.

Mémoires, I, 89.

19 Cr. Mémoires, I, 90: "Feu M. de La Mettrie a fait de l'Irritabilité la base du système qu'il a proposé contre la spiritualité de l'âme [L'homme machine, 18, 22]; après avoir dit, que Stahl \& Boerhaave ne l'avoient pas connue, il a le front de s'en dire l'inventeur; mais je sais par des Vnyes sûres, qu'il tenoit tout ce qu'il pouvoit savoir là-dessus, d'un jeune Suisse, qui, sans être medecin, \& sans m'avoir jamais connu, avoit lu mes ouvrages, \& vû les expériences de l'illustre M. Albinus; c'est là-dessus que la Mettrie a fondé ce sisteme impie, que ses expériences mêmes servent à refuter".

Cf. Richard Toellner, Albrecht von Haller.

21 Thomas S. Hall, "On Biological Analogues of Newtonian Paradigms", Philosophy of Science, 35 (1968), 6-27.

Mémoires sur la nature sensible et irritable des parties du corps animal, par M. Alb. de Haller (Lausanne: M.M. Bousquet (-S. d'Arnay), 1756-1760), I, 7-8.

23 Mémoires, I, 9 (Haller se réfère ici à la section 301 des Institutiones medicae de Boerhaave).

24 Cf. Mémoires, I, 255 et Mémoires (Housset), II, 413-414.

25 Cf. Mémoires, I, 78-79: "L'irritabilité est-elle différente de toutes les autres propriétés du corps? C'est ce que je prouverai très aisément. L'élasticité, qui est celle qui paraît avoir le plus de rapport avec elle, en diffère presqu'en tout. 1. Elle appartient aux fibres sèches, et dans cet état elles n'ont plus aucune irritabilité: on peut s'en convaincre en séchant une grenouille. 2. L'élasticité est une propriété des corps les plus durs, et l'irritabilité des corps les plus souples. Le polype est si irritable que la lumière l'affecte sensiblement quoiqu'il n'ait point d'yeux. Les animaux gélatineux et bien éloignés de toute élasticité le sont beaucoup. M. Whytt ajoute que le mouvement du coeur cesse spontanément et recommence de même, ce qu'on n'observe dans aucune fibre élastique, et qu'en piquant de l'acier avec une aiguille on n'y produit aucune irritation. Guillaume Battie fait observer que l'irritabilité est plus petite dans les vieux sujets que dans les jeunes, quoique les fibres des vieillards soient plus élastiques que celles des enfants". 
Robert Whytt, An Essay on the Vital and Other Involuntary Motions of Animals, 2e édition (Edinburgh: Printed for John Balfour, 1763); cf. en particulier, sect. $X$, "Of the reason why the muscles of animals are excited into contraction by stimuli."

Mémoires, IV, 92.

Elementa physiologiae corporis humani, IV (Lausanne: François Grasset, 1762), p. 462: Haller précise dans la note $(t)$ correspondante quelles furent ses premières étapes dans la conceptualisation de l'irritabilité. Cf. également Mémoires, I, 87-88.

Mémoires, I, 89.

Mémoires, I, 82.

31 Newton, Opticks, Book III, query 31 (New York: Dover), pp. 401-402.

Cf. R. Toellner, Albrecht von Haller, pp. 142 sq.

Mémoires, IV, 25.

34 Mémoires, I, 7: "... ils sont allés jusqu'à prendre cette propriété de nos fibres pour base d'un nouveau système de l'oeconomie animale et en ont déduit les fonctions des vaisseaux, des nerfs, des muscles, en un mot de tous nos organes. L'on peut s'en convaincre en jetant les yeux sur le discours que l'illustre M. J.F. Winter prononça à Franeker en 1746, sur la Dissertation de M. Lups De irritabilitate, et sur celle de MM. Magni et La Motte, dans laquelle ils concluent que toutes les maladies dépendent de l'augmentation ou de la diminution de l'irritabilité des vaisseaux (Ergo a vasorum aucta aut diminuta irratabilitate omnis morbus), système qui revient à peu près à celui qu'ont soutenu MM. Kruger, Nicolai, Whytt, Delius et quelques autres grands physiologistes qui regardent les sensations comme cause de tous les mouvements".

Mémoires, I, 5.

Mémoires, I, 34-35: "J'avais beaucoup travaillé sur l'irritabilité, et surtout sur celle du coeur, j'étais tenté vivement d'en étendre l'empire et d'en faire dépendre le mouvement des artères, celui des glandes, les sécrétions et les fièvres, j'étais prêt d'en faire le ressort unique de la machine animée. On sait assez combien l'homme aime donner de l'importance à ce qu'il a découvert, il voudrait naturellement conquérir l'univers pour ses opinions et établir la monarchie universelle pour l'hypothèse dont il se croit le créateur. Descartes régissait le monde par l'éther et par les tourbillons, et bientôt on a voulu le gouverner par l'attraction. J'avais beau jeu, j'aurais eu pour moi tous ceux qui se plaignent des limites que je donne à l'irritabilité. Les Whytt, les Delius, les Krause, tant d'autres de mes adversaires seraient devenus mes alliés... [Mais 
comme l'expérience ne montre pas que les artères soient vraiment irritables] repoussé par la province que je voulais conquérir, je fus obligé de me replier et de me contenter de l'empire des muscles, assuré à cette irritabilité dont on m'attribuait les progrès".

37 Mémoires, II, 156.

38 Mémoires, I, 297. 